\title{
Optimal rescheduling for the mixed passenger and freight line
}

\author{
B. Davydov ${ }^{1}$, B. Dynkin ${ }^{1} \&$ V. Chebotarev ${ }^{2}$ \\ ${ }^{1}$ Far Eastern State Transport University, Russia \\ ${ }^{2}$ Computer Centre, Far Eastern Branch, \\ Russian Academy of Science, Russia
}

\begin{abstract}
The schedule of the double-track railway line with mixed traffic has two sections, such as passenger and freight segments. Punctuality is a measure of the quality of the passenger traffic. Local traffic punctuality in freight train traffic is an insignificant indicator. The level of the railway operator's expenditure is a more important criterion when the flow of freight trains has passed. We propose a new approach to the problem of operational management, which is based on the direct economic assessment of the dispatcher adjustments.

The method of the operative rescheduling of freight train flow prescribes that the intraday period is divided into the economic and the intensive segments. The criterion for traffic separation into segments is dependent on the level of knock-on delays, respectively, the amount of energy and additional expenditure losses. Cost-effective technology of the local management is used when the number of freight trains is reduced.

In this paper, we consider the total number of secondary unscheduled stops as a measure of the quality of the freight trains flow. This criterion is used together with a specialized stochastic model. The model is based on the composition of the probability distributions of departure times from the original station and the duration of the primary train delays.
\end{abstract}

Keywords: freight train traffic, rescheduling, knock-on delays, stochastic model.

\section{Introduction}

Many rail lines in Eastern Europe, Asia and America are characterized by intense mixed freight and passenger traffic. Operative traffic management in periods when the flow of freight trains passes is significantly different from that which is 
present when a group of passenger trains is moved. When occasional delays occur, the provision of local unconditional punctuality of freight trains is not necessary, i.e. their arrival times at the check points (stations). You only need to exclude the effect of the knock-on to reduce the losses of railway capacity and operational costs. It is not necessary to follow the schedule with high accuracy on each section, since there are the biggest margins to the running and dwell times of freight trains. We understand that you only need to guarantee the macropunctuality of freight trains that are the exact time of arrival at the destination, where the consignment is delivered.

The intensity of the flow of freight trains undergoes a significant change from one hour to another. The reasons for this are that the variations in the freight traffic volume and technology features of the railway operator, as well as planned and unplanned interruptions in the movements. There are additional reserves, primarily, time, which should be used to improve the efficiency of traffic on sites and processing at stations in intervals when the trains' flow decreases. When the flow rate increases, the nature of operational dispatch management must change towards ensuring the passage of the maximum possible number of trains, even with the operating cost overruns. Described kinds of the operating mode may be, respectively, characterized as the economy (cost-effective) and the intensive operational management. In this paper the problem of the dynamic train flow rescheduling in such full formulation is considered for the first time.

It is generally believed that the movement of trains on the railway should be implemented strictly in accordance with the operational schedule. The expected real approach of trains and limitations, associated with the repair and maintenance work of the infrastructure, are taken into account when planning of the freight segment. In practice, there are a large number of violations of the schedule due to technical and technological failures. Optimal dispatching suggests that you need to make adjustments in the schedule for the real specific scenario, which reduces the number of conflicts or damage caused by their appearance.

In most published work there are solutions to the problem of efficient restructurings of the passenger segment of traffic. Those solutions are compared in total delay, which acquire all trains for a given period of time. Local punctuality in the freight flow movement recedes into the background. Here you want to use other criteria, adequate economic interests of the company - train traffic operator. The analysis shows that as a criterion the operating costs for passing trains or part of the profits may be used, which corresponds to the freight trains traffic on the railroad section [1]. Formulation of the economic criterion is made in section 4.

When searching for the best solutions to online rescheduling used deterministic or stochastic approach to the description of the present situation and its further development. Deterministic approach assumes that there is full information of the train's arrival and departure schedules at boundary stations of the section, actual (and constant) characteristics of rolling stock and infrastructure. 
The stochastical model assumes that there are only probabilistic characteristics of the incoming streams and random delays during the trains' movement on the scheduling of freight train flow. Section 5 of this paper attempts to use both models, such as stochastic model of train traffic and deterministic energy consumption model, when developing the algorithm of the optimal dispatching decisions. We show the first phase of the algorithm defines boundaries between periods of economy and intensive modes of the freight trains management.

In this case the data on the expected arrival times of trains and the technical condition of infrastructure are the original information. These data defines the frequency and the localization of non-scheduled delays. The totality of data of these latter species we'll call the scenario of the rail section functioning. In this paper (Section 6), we study the problem of segmenting a dense flow of freight trains following the same path of the double-track railway line. The solution to this problem is based on a stochastic predictive model of unscheduled stops.

The second stage of the algorithm solves the optimization problems of various types, respectively for each of the foregoing periods. The energy-optimal trajectories for the trains that move at a time when the cost-effective mode works, are determined on the basis of a deterministic model of the traction energy consumption. In this problem, looking for an optimal distribution of the surplus time that is added to the scheduled running time at the elements of railway section during targeted adjustment of train traffic. It was determined by analyzing the structure of the costs for the trains movement on a rail section, that implementation of energy optimality is a necessary condition to ensure minimum operational costs [2]. Scheduled (mean) departure interval for the intensive segment of the train flow is calculated from a given level of the throughput degradation because of disruptions. The calculation is based on the known statistic of a technical failure flow (non-scheduled delays). All variants of the stops distributions are obtained from analysis of the historical data. Required sample is selected from the available set in accordance with the projected scenario.

Section 7 presents data on the effectiveness of the implementation of the methodology proposed in this study. There are evaluation of the economical gain in this study, that is obtained when the operational scheduling and rescheduling of train traffic is used. These results were determined by experimental testing of the elements of adaptive dispatching at the some sections of the Trans-Siberian main rail line (Russia). The final section 8 we conduct the discussion of the results and the prospects for further research.

\section{Literature review}

Most of the published work on the problem of operative rescheduling of trains for situations where disturbances occur is based on deterministic models of the functioning of the railway. The main types of problems which are solved in the process of searching for optimal adjustments are conflict detection and resolution (CDR) and train speed coordination (TSC) [3-6]. In these problems, 
the best order of trains movement determined on the condition that arise the deviations from their schedule (delays), which lead to conflicts. Most of the works explores the algorithms corrections of the passenger train traffic on busy lines. Typically, it is considered the total value of delay of all trains in a given period. Authors, that attempt to use economic indicators of quality of passing the passenger flow, one way or another interprets the volume of delays. The decisions for rescheduling, which minimize delays of the trains, are calculated by constructing the discrete models of movement and use of integer or partially integer linear programming (for example [7, 8]).

The authors do not know any studies that use explicit economic criteria in solving the local optimization problem of the railway traffic. This criterion reflects the amount of profit or loss. In some papers $[9,10]$ only the idea of operational management is offered, which improves the transfer and handling of freight trains, with direct examination of the profit or the loss. A similar problem is formulated for the area of road freight haulage [11]. There are a limited number of publications, which show the decisive role of the energy resources in the formation of operating costs, which depend on the mode of operation. Many papers have been published (e.g. $[12,13])$, which address to the construction the trajectory of the train, providing a minimum consumption of energy. Rational allocation of the extra time between the elementary path segments is provided by the mechanism of the timetable adjustment that aims to fuel economy.

When ignoring the risk of random perturbations, there is a primary drawback of deterministic models that are used to find rational solutions. This drawback is largely compensated by using stochastic models in addition to deterministic ones. Forecasting of the uncontrolled oscillations of speed and stops, as well as their distribution along the chain of trains gives the ability to assign rational adjustments and simulate their likely consequences. One of the first papers considering this problem [14] proposes analytical method for the determination of delays by a convolution of the initial (inlet area) and delays arising again. The analysis is limited because it uses the assumption of uniform distribution of secondary delays. In reality, this assumption is not confirmed.

There is a well-known model [15] of the accumulation of trains' delays on the railroad, which uses a probabilistic approach. This model considers the total running time as sum of the partial intervals of the moving trains at the elements of section. Each interval is considered as a random variable, and this quantity is usually taken with the exponential distribution. The model performs the multiple numerical simulation of motion with the small headways of a pair of trains, resulting calculated the mathematical expectation of the running time. Deceleration of the first train on some sections leads to the knock-on delay, which leads to the late arrival of the rear train to the final station. This work does not consider the probability distribution function of the headways at the exit of rail section and the mechanism of the delays propagation through the chain of trains. This feature is not possible to get an estimate of the Intertrain intervals, corresponding to the given probability of non-scheduled stops. In this paper an attempt is made to eliminate the weaknesses of these statistical models. 


\section{Preventive adjustment of the freight trains traffic}

Capacity of most main double track lines with mixed traffic does not exceed one hundred pairs of trains a day. The volume of freight traffic on such lines is a maximum of 60-70 pairs of trains. Under such traffic intensity, the averaged value of period of the schedule is 15 minutes. This value is significantly higher than the minimum headway that you want to provide in the freight trains' flow for the safety. So, when the heavy trains (up to 8000 tons) follow, the minimum estimated time interval is 4 minutes. This interval is obtained for a standard three-valued signalling system and the length of the block section, equal to $2 \mathrm{~km}$ (Russian Railways). This value determines the theoretical limit of dynamic rapprochement, when the impact to rear trains of front train begins.

The studies suggest that on the sections of main rail line usually occurs at least two periods of the strengthened freight traffic (Figure 1). During these periods, the intensity of the flow of train's $n$ increases by $1.5-2$ times. This leads to the fact that the processes of the appearance and reproduction unscheduled delays becomes more powerful. This process is accompanied by a decrease in the average train speed, and the rail line capacities. Traffic control in this period can be only intense, that is using the acceleration mode (i.e. replenishment of running time) to compensate random delays.

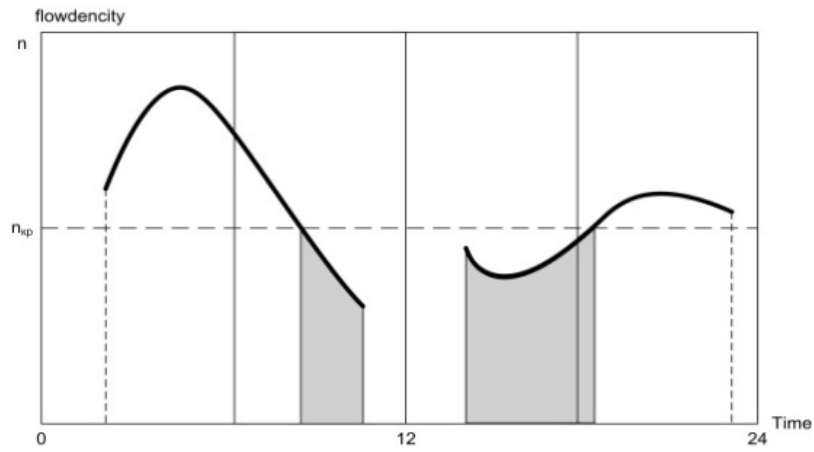

Figure 1: Trains' flow density during the day.

During the period when the flow rate is small, there is the possibility of using additional reserves of time for increasing efficiency in the movement of trains. Extra time, which is introduced in the schedule, can be used in two ways:

- by increasing the running time of each train on the elements of the rail section and on the whole route;

- by storing the trajectory (i.e., the each line of schedule) unchanged with the increasing headway.

It has been shown [2] that the main component of the gain of economic result of the railway operator, is to reduce the consumption of fuel and energy resource. Traffic control, which is aimed to obtain such a result, can be called economical. As mentioned earlier, train manager has a fairly wide range of freedom to choose 
the nomenclature of freight trains, the order and the schedule of their movements, because there is no need to provide a high level of micropunctuality.

Thus, the preventive measures that manager can be used in the on-line work with freight trains in order to reduce costs are divided into two groups:

- the appointment of such a number and nomenclature of freight trains, which provide the maximum economic result;

- the appointment of such the running times on the elements of the path, which provides minimum power consumption, and therefore operating costs (i.e., micro-management).

The optimized schedule for the period, when using cost-effective management is introduced into the work by manager while reducing the intensity of the incoming flow of trains below a certain level $n_{c r}$ (see shaded area in Figure 1). The optimized schedule for the period when is used the cost-effective management, is introduced into the work by manager when the intensity of the incoming flow of trains below a certain level is reduced (see shaded area in Figure 1). Values of the corresponding headways $T_{\text {dep }}$ (or throughout the flow) and the number of knock-on delays $M_{\text {knock }}$ depend on the duration of nonscheduled stops and on the nature of their probability distributions. The latter characteristic is based on statistics of the failures, which obtained in previous periods of operation of the particular section of railroad. Section 5 discusses the following question: what is a set of models and methods to be used when determining the optimal solutions for the on-line management of the freight trains flow. The problem of determination of the critical value of average headway is discussed in Section 6. The critical value is the boundary between the economic and intensive modes.

\section{The criterion for optimal control of the freight trains traffic}

Operational management system is initially oriented in the direction that traffic safety has the highest priority. The criterion of capacity becomes very important in the periods that are characterized by an increase in the demand for large volumes of passengers and freight transportations. In the prior studies some researchers attempted to use the integral economic criteria in the operational management of the railway. For example, in [16] proposed a method of search of optimum solutions to the effective movement of container trains on the transcontinental route. There is regarded the problem of ensuring of such a train traffic, which gives a minimum total loss $\Delta I_{\text {pen }}$ of the operating company, which occur due to the penalties for late arrival of trains at the destination. The described approach should recognize the limited, since management personnel in this case there is no incentive to further increase economic gain ( $\Delta B=\Delta I-\Delta E)$ if the costs already reached the level of $D$.

We suggest a new economic efficiency criterion, which is used to find the decisions for the operational management of the trains' traffic. It is helpful to use 
the increment of the local profit $\Delta B$ as the criterion for assessing the quality of the traffic manager's decisions, who controls the movement of freight trains on the section [1]:

$$
\Delta B=\Delta I-\Delta E
$$

where $\Delta I$ is the change of an operator company's income because of the realized adjustment; $\Delta E$ is the additional expenses for making the adjustment.

Optimal variant is chosen from the range of feasible decisions according to the maximum of the objective function: $\Delta B \rightarrow \max$.

The process of forming the economic result all through following of freight train on the route can be displayed graphically (see Figure 2). The model reflects the dynamics of profit of the operating company in those intervals when the train travels along the sections and is handled at the stations.

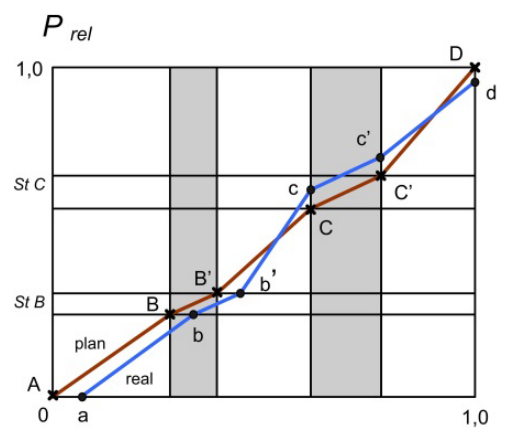

a)

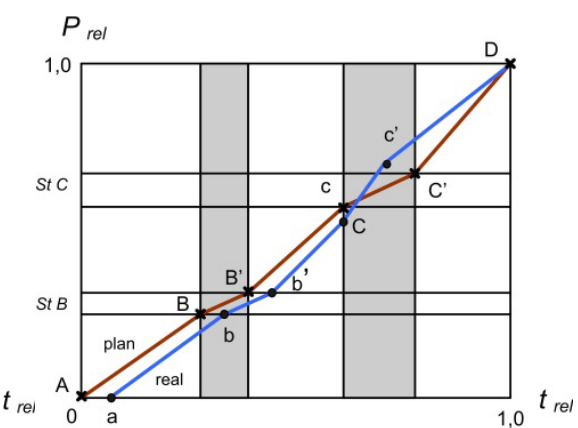

b)

Figure 2: The diagram of formation of the result of freight transportation in accordance with the planned and actual schedules: a) the case of deterioration of the planned economic result; b) the recovery of planned result by performing the rational adjustment.

This example illustrates the fact that the total profit decreases (point $d$ in Figure 2a) with respect to the planned value (point $D$ ). The economic loss occurs because of overcoming the obstacles, although it is retained punctuality of arrival of the train at the destination. The loss of profit, which arose on the segment $A C$ (see Figure 2b) is able to make up for the case, when there is scope for the implementation of the adjustment measures. For this purpose priority processing of the train at the station $C$ and it energy efficient movement on the section $C D$ be organized. But, it is necessary disrupt the scheduled working intervals.

\section{Comprehensive model for the problem of finding the optimal dispatching decisions}

We assume that the flow of freight trains moves along the one path of the double-track rail section. The trains go exactly according to the prescribed schedule, unless there are the occasional delays. The reserve of time is included 
in the schedule, which is usually used to increase the normative interval. The additional buffering times absorbs the random deviations, thereby preventing the spread of the knock-on delays in the chain of trains. The headway increases in the period of time, when moves a weak flow of trains. Train dispatcher is to decide the Problem I, in what period should be implemented the intensive management mode otherwise the economy mode control. This value of the headway $\tau_{\text {сг }}$ is the boundary between these periods in which the probability of unscheduled delays $P\left(\tau_{\text {del }}\right)$ doesn't exceed the predetermined value.

The initial problem of determination the optimal solutions to control the flow of freight trains is divided into two subsequent, after determining the specified periods. Operative dispatching control in intensive period must be organized in such a way as to get the best economic result. It is formulated as the Problem IIa. Optimal solution of the problem is the maximum income that is obtained by the implementation of the priority departure the most "expensive" trains in the considered planning period $T$. The worth of freight train it is the value of potential income given the likely penalty for delay in freight delivery. The stochastic model, which is used in solving the problem, similar to the model that is proposed for operational control of information packets in a communications network [17].

During the weak movement it appears the extra time, that the dispatcher can use for the targeted increase the running time of trains on the section. This ensures the most efficient use of the energy that is consumed for the traction. Energy model of the train, which is used in the optimization problem IIb, belongs to the class of deterministic models. When considering the criterion of energy efficiency, the problem of optimal allocation of additional running time resolves using dynamic programming (see for example, [12]) or effective heuristics [13].

\section{Modalities for the introduction of the economic mode of train traffic}

In this section we examine the question of what value of the headway you have to select in order to provide the minimum number of knock-on delays on the railway line. Obviously, if we increase this interval, it will cause less impact of the one train to another one that goes behind the first in the same direction, and the amount of delays will fall. If the flow of the trains becomes weaker the average value of the interval to be observed at the station of departure is reduced. Let us find the limit value of the headway at which it will be possible to use the economy mode effectively.

Consider the following scenario. Several trains move from the Station $A$ to the Station $B$. The actual departure interval equals $T_{\text {dep }}$. First train is experiencing the delay on its way. The initial delay causes the knock-on stops of the following trains because there is the effect of mutual influence of trains and small intervals are available. The certain time and energy is lost at each stop. 
Consider the problem of propagation of a nonscheduled small stop of the duration $\tau_{1}$, when there is a dense flow of trains on the rail section $A B$. We assume that the random variable $\tau_{1}$ has the density function $g(t)$. In formulating the problem it is assumed that all trains are physically the same, i.e. have identical dynamic characteristics. Also it is considered the characteristics of the scattering length of primary stop do not depend on the place of its occurrence.

In prior works the various models are discussed which describe the functions of the frequency and length of nonscheduled delays. Thus, in the papers $[15,18,19]$ the exponential probability distribution is used as a stochastic model of the train movement, which is violated, when "internal" reasons occur. We can see that the distribution of the secondary delays which are caused by the mutual influence of the late trains, is obtained as the composition of individual distributions both the stops and headways.

When constructing the model we assume that following trains are stopped with the red signal at a minimum safe distance of $S_{0}$. In accordance with the algorithm of the signaling system and the applicable rules, the rear train starts to move immediately after the front train resumes the motion. Obviously, the headways of the trains that are undergone to knock-on delays, on the rest of the way are equal to $T_{2}^{a}=T_{3}^{a}=\ldots=T_{0}$ (see figure 3).

Let $T_{\text {dep }}$ is defined as the minimum interval departure of trains from the station $A$, under which the probability $\mathrm{P}\left(M_{\text {knock }} \geq k\right)$ that the number of secondary stops $M_{\text {knock }}$ is not less than $k$, does not exceed a specified level $\alpha$ .We must take into account in the calculation, the minimum time interval $T_{0}$ of trains' pass, which is set in accordance with the requirements of traffic safety, is determined by the rate $v_{0}: T_{0}=\frac{s_{0}}{v_{0}}$.

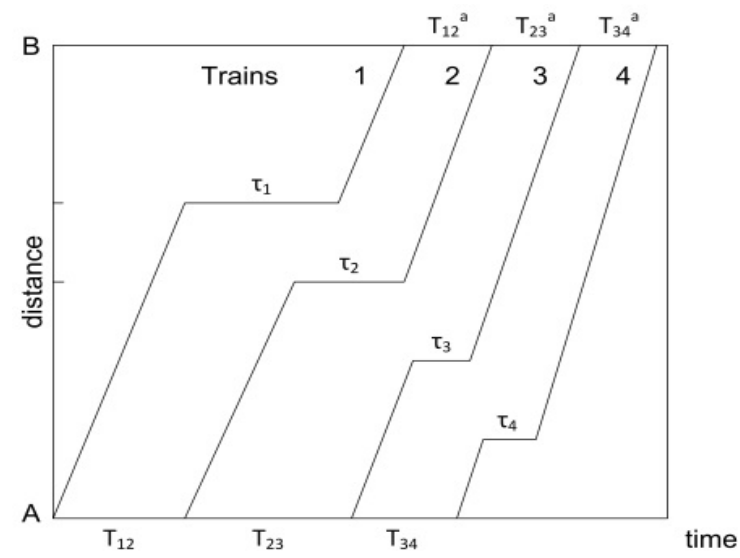

Figure 3: The diagram of the knock-on delays. 
Express headway $T_{\text {dep }}$ as the sum of the unknown parameter $T$ and a fixed amount of the safe interval $T_{0}: T_{\text {dep }}=T+T_{0}$. Considering the trajectories of movement of trains as the geometric elements, we obtain the following:

$$
M_{\text {knock }}=k \Leftrightarrow k T<\tau_{1}<(k+1) T, \quad 1 \leq k \leq n-2,
$$

where $n$ is the number of trains. Consequently,

$$
M_{\text {knock }} \geq k \Leftrightarrow \tau_{1}>k T
$$

Then,

$$
P\left(M_{\text {knock }} \geq k\right)=P\left(\tau_{1}>k T\right)=\int_{k T}^{\infty} g(t) d t, \quad 1 \leq k \leq n-1
$$

Let us denote by $x_{q}$ the $q$-quantile of the random variable $\tau_{1}$. Then $x_{1-\alpha}$ is the root of the equation:

$$
\alpha=\int_{x}^{\infty} g(t) d t
$$

with respect to the variable $x$. Equating $k T$ to $\alpha$, we get the formula:

$$
T=\frac{x_{1-\alpha}}{k}
$$

Thus, the headway $T_{\text {dep }}=T+T_{0}$, for which the probability of occurrence of not less than $k$ secondary stops, does not exceed a predetermined level $\alpha$, is evaluated by the value $T+T_{0}$, where the time $T$ is determined by Equation (6). This value is treated as a boundary that separates periods of the economic and intensive mode of traffic at the rail site. These periods shall be appointed on the basis of analysis of predictive information about the number of trains that are planned to be sent to the section in the coming every hour.

\section{Experimental results and their interpretation}

On a section of X-B with the length of 210 kilometers in the eastern part of the Trans-Siberian Main Railway, there is an area where often there is the tension (segments 9-10, the total length of 19 kilometers). At the period when experimental studies were carried out, short nonscheduled stops which caused by the knock-on effect, regularly appeared in this area. We got 52 values of "primary" stop duration, which is a sample of the distribution of random 
quantity $\tau_{1}$. Hypothesis was confirmed using Pirson criterion that distribution $\tau_{1}$ is exponential with the parameter $\lambda=0.261 / \mathrm{min}$.

The histogram and the hypothetical density function are depicted in Figure 4. Along the horizontal axis the duration of the primary delay is marked in minutes.

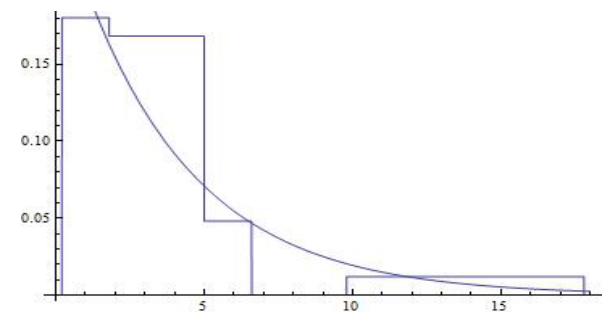

Figure 4: The histogram and the hypothetical density function.

It was shown the consistency on a significance level of 0.05 of the above hypothesis with the experimental data. More precisely, we shall indicate that the value of chi-squared statistics equals 8.89. At the same time the critical criterion value, which is found using the chi-square distribution with four degrees of freedom, equal to 9.49 .

As an example of the use estimated duration of interval of departure $T_{d e p}$ consider the following scenario. It is expected increase in the flow density of freight trains on the railway section. We expect that the number of delays does not exceed four with a probability less than 0.9 . By solving the equation with $g(t)=\lambda e^{-\lambda t}, t>0$, we find that $x_{1-\alpha}=\frac{1}{\lambda} \ln \frac{1}{\alpha}$. By the hypothesis it is given, that $\alpha=0,1, \lambda=0,26$, hence we obtain: $x_{0,9}=8,86$.

Then, the calculated value $T$ using formula (6) when $k=5$ is equal to 1.77 min. Taking into account that safe headway is 4 minutes we conclude that departure interval from the original station should be at least 5.8 minutes. Experimental results show that the average length in reality, when condensed flow of freight trains, is equal to 8 minutes.

\section{Conclusions and future work}

In this study, we determined that a significant improvement in the economic performance of railway in freight traffic can be obtained if we target and apply the on-line rescheduling. This operation is to that the flow of freight trains is divided into two segments such as economy and intensive, when the short-term plan is produced. Then in each segment is set a specific mode of the freight trains movements. It is allowed a slight increase in the scheduled running time of the train during the implementation of the economy mode. The time margin is used to reduce the consumption of energy resources on the some elements of the path. 
In this paper we developed a statistical model that allows the dispatcher to reasonably assign periods of use the economy and intensive modes of the freight traffic. Obviously, improving the management of trains is not exhausted with using the methodology proposed in this paper. The increase of speed and accuracy of the system sending commands to control the train locomotives also reduces the knock-on effect and hence improves the economic result. We believe this line of work is very promising and should be subject of enhanced development.

In further studies, we expect to improve the quality of the stochastic model of trains' unscheduled stops by the addition to the variable running time on the elements of path. In addition, we plan to develop a method that allows predicting difficulties in the movement of trains associated with the repair and maintenance works, the deteriorating weather and with other circumstances.

\section{Acknowledgements}

This work was supported by Russian Foundation for Basic Research, under research project № 12-08-98513-p_vostok_a. Thanks are due to referees for their constructive comments.

\section{References}

[1] Давыдов, Б. И., Модель формирования экономического результата при движении грузового поезда. Экономика железных дорог, 9, pp. 62-65, 2012.

[2] Давыдов, Б. И. \&Котлярова Е. В., Экономическое обоснование рациональной скорости движения поездов. Экономика железных дорог, 4, pp. 17-23, 2008.

[3] D'Ariano, A., Pacciarelli, D. \& Pranzo, M., A branch and bound algorithm for scheduling trains in a railway network. European Journal of Operational Research, 183 (2), pp. 643-665, 2007.

[4] Quan Lu, Dessouky, M. \& Leachman, R. C., Modeling Train Movements through Complex Rail Networks. ACM Transactions on Modeling and Computer Simulation, v. 14. pp. 32-76, 2004.

[5] Törnquist, J., Computer-based decision support for railway traffic scheduling and dispatching: A review of models and algorithms. Proceedings of ATMOS, Palma de Mallorca, Spain, October 2005.

[6] Carey, M. \& Crawford., I., Scheduling trains on a network of busy complex stations. Transportation Research, Part B, 41, pp. 159-178, 2007.

[7] Cordeau, J.-F., Toth, P. \& Vigo, D., A survey of optimization models for train routing and scheduling. Transportation Science, 32 (4), pp. 380-404, 1998.

[8] Huisman, D. Kroon, L., Lentink, R. \& Vromans, M., Operations research in passenger railway transportation. Erasmus Research Institute of Management (ERIM). Research Paper № ERS-2005-023-LIS, 2005. 
[9] Törnquist-Krasemann, J. Design of an effective algorithm for fast response to the rescheduling of railway traffic during disturbances. Transportation Research Part C, Dec. 16 ${ }^{\text {th }}, 2010$.

[10] Mes, M., van der Heijden, M., van Harten, A. Comparison of agent-based scheduling to look-ahead heuristics for real-time transportation problems. European Journal of Operational Research, 181(1), pp. 59-75, 2007.

[11] Shah, N., \& Brueckner, J. K., Price and Frequency Competition in Freight Transportation. UCTC-FR-2011, University of California, pp. 15-38, 2011.

[12] Кузнецова, А. А., Оптимизачия технологии вождения грузовых поездов по перегонам по критерию минимума эксплуатациинных расходов по пробегу. Дис. к.т.н., Новосибирск, р. 152, 2006.

[13] Bocharnikov, Y.V., Tobias, A.M., Roberts, C., Hillmansen, S. \& Goodman, C.J., Optimal driving strategy for traction energy saving on DC suburban railways. IET Electr. Power 1(5), pp. 675-682, 2007.

[14] Muhlhans, E. Berechnung der Verspatungsentwicklung bei Zugfahrten. Eisenbahntechn. Rundschau ETR, 39 (7/8), pp. 465-468, 1990.

[15] Carey M., \& Kwiecinski A., Stochastic approximation to the effects of headways on knock-on delays of trains. Transportation Research Part B 28 (4), pp. 251-267, 1994.

[16] Ivnitsky, V. A., Non-Observance Risk Identification of the Prescribed Average Daily Speed Level by Speeded-up Container Trains due to Technical Failures. VNIIZDT Bulletin, v. 12, pp. 41-44, 2012.

[17] Bent, R., \& Van Hentenryck, P., The Value of Consensus in Online Stochastic Scheduling. In: Proceedings of the ICAPS, pp. 219-226, 2004.

[18] Vansteenwegen, P., \& Van Oudheusden, D., Decreasing the passenger waiting time for an intercity rail network. Transportation Research Part B: Methodological, Vol. 41, Issue 4, May, pp. 478-492, 2007.

[19] Ho, T.K. \& He, Y.Q., Evaluation on service quality of railway timetable.

[20] Traffic and Transportation Studies: Proc. of the 6-th International Conference on Traffic and Transportation Studies, Nanning, China, 2008. 